\title{
Repeated positive acceleration exposure exacerbates endothelial dysfunction in high-fat-diet-induced hyperlipidemic rats
}

\author{
Zhihui Yang ${ }^{1}, \mathrm{Hua} \mathrm{Ge}^{2}$, Chunya Wang ${ }^{3}$, Zhongdong Liं ${ }^{1}$ Qingjun Zhang ${ }^{1}$, Jianchang Wang ${ }^{4}$
}

1Department of Pharmacology, General Hospital of PLA Air Force, Beijing, China
${ }^{2}$ Aviation Physiological Laboratory, Institute of Aviation Medicine, PLA Air Force,
Beijing, China
${ }^{3}$ Emergency \& Critical Care Center, Beijing Anzhen Hospital, Capital Medical
University, Beijing, China
${ }^{4}$ Center of Clinical Aviation Medicine, General Hospital of PLA Air Force, Beijing, China

Submitted: 10 December 2016

Accepted: 29 January 2017

Arch Med Sci 2017; 13, 4: 937-946

DOI: https://doi.org/10.5114/aoms.2017.68144

Copyright $\odot 2017$ Termedia \& Banach

\section{Abstract}

Introduction: It remains unclear whether exposure to repeated positive acceleration $(+\mathrm{Gz})$ can exacerbate endothelial dysfunction on the basis of hyperlipidemia. The aim of this study was to investigate the effect of repeated $+\mathrm{Gz}$ exposure on endothelial function in high-fat-diet-induced hyperlipidemic rats. Material and methods: Male Sprague-Dawley rats were randomly divided into control, repeat $+\mathrm{Gz}$ exposure, high-fat diet (HFD), and $+\mathrm{Gz}+\mathrm{HFD}$ groups. The rats in the $+\mathrm{Gz}$ group were exposed to $+\mathrm{Gz}$ and the rats in the HFD group were fed a diet with $2 \%$ cholesterol. The rats in the $+\mathrm{Gz}+\mathrm{HFD}$ group received both the $+\mathrm{Gz}$ exposure and HFD. Eight weeks later, the endothelium-dependent relaxation of the aorta was tested and the ultrastructure of the endothelial cells was observed using transmission electron microscopy. Quantitative real-time polymerase chain reaction and Western blot were used to detect the mRNA and protein expression of endothelial function-associated proteins.

Results: Repeated $+\mathrm{Gz}$ exposure elevated the serum level of LDL-C in HFD rats. In the $+\mathrm{Gz}+\mathrm{HFD}$ rats, the ACh-induced relaxation in the aorta rings was significantly attenuated and the endothelial cells of the aorta were dramatically damaged compared with HFD rats. Nitric oxide content and eNOS expression in the aortic tissue were markedly decreased and the oxidative stress was more serious in the +Gz + HFD rats compared with HFD rats. In addition, repeated $+G z$ exposure significantly increased serum ox-LDL level and LOX-1 expression in the aorta of HFD rats, thereby activating NF- $\mathrm{BB}$ p65 and upregulating the expression of interleukin 6, ICAM-1 and VAP-1.

Conclusions: Repeated $+G z$ exposure promotes endothelial dysfunction in HFD-induced hyperlipidemic rats.

Key words: positive acceleration, endothelial dysfunction, hyperlipidemia, high-fat diet.

\section{Introduction}

Aircraft pilot is a special occupation which may constantly expose the person to various abnormal environments including positive acceleration $(+G z)$ load. During execution of aerial tasks, the $+G z$ in the head-to-foot

\author{
Corresponding authors: \\ Jianchang Wang \\ Center of Clinical \\ Aviation Medicine \\ General Hospital \\ of PLA Air Force \\ No. 30 of Fucheng Road \\ Beijing 100142, China \\ Phone: +86 1066928001 \\ E-mail: kzdw66@126.com \\ Chunya Wang \\ Emergency \& Critical \\ Care Center \\ Beijing Anzhen Hospital \\ Capital Medical University \\ No. 2 of Anzhen Road \\ Beijing 100029, China \\ Phone: +861084052201 \\ E-mail: drwangchunya@ \\ 163.com, \\ yanjiu999999@sina.com
}


direction generated by the accelerating aircraft is characterized by high $\mathrm{G}$ values, long in duration, and repeated occurrence [1]. Repeated $+\mathrm{Gz}$ exposure strongly promotes blood flow toward the lower body, which tends to reduce cerebral, coronary and renal blood flow, resulting in severe damage to multiple organs [2-4]. Although the development of anti-G equipment improves the $+\mathrm{Gz}$ tolerance of pilots [5], the harmful effects of $+\mathrm{Gz}$ exposure on pilots cannot be eliminated. Therefore, to better understand the underlying mechanisms of the pathological process of the damage induced by repeated $+\mathrm{Gz}$ exposure would be beneficial to avoid the related diseases in pilots, thereby prolonging the service years of pilots.

Besides the typical symptoms of loss of consciousness and visual function, which were reported by numerous studies [6-8], repeated +Gz exposure-induced cardiovascular diseases are also a key factor that threatens pilots' health. A previous study by Martin et al. reported that repeated high $+\mathrm{Gz}$ and the methods to prevent loss of consciousness cause stress on the cardiovascular system [9]. Atherosclerosis (AS) is a common cardiovascular disease that is characterized by intimal plaques and can lead to relative diseases throughout the vascular system such as coronary artery disease, stroke and peripheral artery disease. Hyperlipidemia is the main risk factor for AS [10]. During flight maneuvers, $+\mathrm{Gz}$ load induces stress responses and disorders of the neuroendocrine system, which may have adverse effects on lipid metabolism. Luo et al. found that $+\mathrm{Gz}$ exposure promoted a metabolic disorder of serum lipid in rabbits fed with a high-cholesterol diet [11], which indicated that $+\mathrm{Gz}$ may accelerate the progression of AS.

Endothelial cell dysfunction is an important contributor to the pathology of AS [12, 13]. Endothelial dysfunction is characterized by a reduction of the bioavailability of vasodilators and increase of endothelium-derived contracting factors, which leads to endothelium-dependent vasodilation impairment [14]. Endothelial cell dysfunction can be induced by numerous factors including oxidative stress, proinflammatory cytokines, disturbed blood flow, etc. [12]. Aeromedical studies have found that $+G z$ exposure increased plasma levels of calcitonin gene related peptide (CGRP) and endothelin and reduced superoxide dismutase (SOD) activity and increased malondialdehyde (MDA) levels in serum [15]. Based on these findings, we hypothesized that $+\mathrm{Gz}$ exposure may induce endothelial cell dysfunction and promote the process of AS. In the present study, high-fat diet (HFD)-induced AS rats were used to investigate the effects of repeated $+G z$ exposure on endothelium-dependent vasodilation of the aorta and the production of associated bioactive factors.

\section{Material and methods}

\section{Animals}

A total of 32 male Sprague-Dawley (SD) rats weighing 180-220 g were obtained from the Animal Center of the Academy of Military Medical Sciences (Beijing, China). All the animals were housed at $22 \pm 2^{\circ} \mathrm{C}$ on a $12 \mathrm{~h}$ light-dark cycle and received free water ad libitum in the Centre of Laboratory Animals of the $304^{\text {th }}$ Clinical Department of General Hospital of PLA (Beijing, China) in accordance with the National Institutes of Health Guide for the Care and Use of Laboratory Animals.

\section{Experimental design}

The animals were randomly divided into four groups: (1) control group $(n=8)$ (Con), (2) repeat positive acceleration exposure group $(n=8)$ $(+\mathrm{Gz}),(3)$ high-fat diet group $(n=8)$ (HFD), and (4) $+\mathrm{Gz}+$ HFD group $(n=8)$. The rats in the control and the $+\mathrm{Gz}$ group received standard laboratory food. The rats in the HFD and the $+\mathrm{Gz}+\mathrm{HFD}$ groups received HFD ( $2 \%$ cholesterol) during the period of the experiment. The centrifuge protocol was based on the previous studies with modifications according to our preliminary experiments [16]. Each rat was put into a box fixed on the arm of the centrifuge facing towards the axis of the centrifuge. Rats in the $+\mathrm{Gz}$ and $+\mathrm{Gz}+\mathrm{HFD}$ groups were exposed to $+\mathrm{Gz}$ in an animal centrifuge with $2 \mathrm{~m}$ radius. The peak value of gravity was set at $+10 \mathrm{G}$ and the onset rate was set at $1 \mathrm{G} / \mathrm{s}$. The rotation lasted for $30 \mathrm{~s}$ and was repeated 3 times The interval between 2 rotations was 1 min. Rats in the Con and HFD groups were not exposed to the acceleration. The centrifuge was performed 8 weeks with the frequency of 3 times a week.

\section{Aortic ring assay}

The rat was anesthetized with $10 \%$ chloral hydrate and the thoracic aorta was isolated. The aorta was transferred into Krebs' solution (118.0 mM NaCl, $4.75 \mathrm{mM} \mathrm{KCl}, \mathrm{CaCl}_{2} \cdot 2 \mathrm{H}_{2} \mathrm{O} 2.54$, $\mathrm{KH}_{2} \mathrm{PO}_{4} 1.19, \mathrm{MgSO}_{4} \cdot 7 \mathrm{H}_{2} \mathrm{O} 1.19, \mathrm{NaHCO}_{3} 25$, glucose 10.0, $\mathrm{pH} 7.4$ ) and the surrounding tissue and fat was gently removed. The aorta was cut into 5-mm long pieces and hung between 2 l-shaped stainless steel sticks. One stick was fixed at the bottom of the organ bath and the other connected to a force transducer. The organ bath was filled with $10 \mathrm{ml}$ of Krebs' solution and with a continuing supply of carbogen $\left(95 \% \mathrm{O}_{2}\right.$ and $\left.5 \% \mathrm{CO}_{2}\right)$ at $37^{\circ} \mathrm{C}$. The aortic ring was then allowed to equilibrate in the bath at $1.5 \mathrm{~g}$ for $1 \mathrm{~h}$. The Krebs' solution was refreshed every $30 \mathrm{~min}$ and the tension was readjusted to $1.5 \mathrm{~g}$ if necessary. In the first set of experiments, the aortic ring was pre-contract- 
ed with norepinephrine (NE, terminal concentration: $10^{-7} \mathrm{M}$ ). When the plateau was achieved, the aortic ring was relaxed by cumulative addition of acetylcholine (ACh, terminal concentrations: $10^{-9}$, $10^{-8}, 10^{-7}, 10^{-6}$, and $\left.10^{-5} \mathrm{M}\right)$. In the second set of experiments, the aortic ring was washed using Krebs' solution 3 times, the tension readjusted to $1.5 \mathrm{~g}$, then it was allowed to equilibrate for $1 \mathrm{~h}$. After pre-contraction with NE, the aortic ring was relaxed by cumulative addition of sodium nitroprusside (SNP, terminal concentrations: $10^{-9}, 10^{-8}$, $10^{-7}, 10^{-6}$, and $10^{-5} \mathrm{M}$ ). The changes of tension were recorded by the signaling collecting system (ZH, Zhenghua Bioinstrumentation Co., Ltd, Anhui, China) and concentration-response curves were constructed.

\section{Measurement of serum lipid}

The whole blood was collected from the abdominal aorta and centrifuged at $1,000 \mathrm{~g}$ for $10 \mathrm{~min}$ at $4^{\circ} \mathrm{C}$. Total cholesterol (TC) and triglyceride (TG), high-density lipoprotein cholesterol (HDL-C) and low-density lipoprotein cholesterol (LDL-C) in the serum were measured using an automatic analyzer (Hitachi model 7600 Hitachi High-Technologies Corporation, Ibaraki, Japan).

\section{Transmission electron microscopy}

Ultrastructural alterations of the thoracic aorta of the rats were detected by transmission electron microscopy (TEM). Samples for TEM examination were cut into pieces less than $1 \mathrm{~mm}^{3}$ and immersed in $3 \%$ glutaraldehyde in $0.1 \mathrm{M}$ sodium cacodylate buffer ( $\mathrm{pH}$ 7.4) for $3 \mathrm{~h}$ for primary fixation. After being rinsed using sucrose buffer, the samples were postfixed in $1 \% \mathrm{OsO}_{4}$, dehydrated in ethanol and embedded in Epon 812. Ultrathin sections cut from blocks after staining with uranyl acetate and lead citrate in darkness were examined with a H-7650 transmission electron microscope (Hitachi - Science \& Technology, Tokyo, Japan).

\section{Measurements of oxidative stress and NO content in the aorta}

Samples of aortic tissue were homogenized in cooled PBS on ice and centrifuged at $12,000 \mathrm{rpm}$ for $10 \mathrm{~min}$ at $4^{\circ} \mathrm{C}$. The supernatant was collected and the concentration of protein was determined using a BCA protein assay kit (Nanjing Jiancheng Bioengineering Institute, Nanjing, China). The total antioxidation (T-AOC) and the content of MDA and nitric oxide (NO) in the aorta were determined using indicated commercial biochemical assay kits (Nanjing Jiancheng Bioengineering Institute, Nanjing, China) following the manufacturer's instructions.

\section{ELISA}

Samples of aortic tissue were prepared as above. The supernatant was used for ELISA and the results were standardized by protein concentration. The whole blood was collected and centrifuged at 3,500 rpm for $15 \mathrm{~min}$ and the serum was collected and used for ELISA. The serum levels of oxidized low-density lipoprotein (oxLDL) and soluble intercellular adhesion molecule 1 (sICAM-1) and interleukin (IL)- 6 levels in the aorta of the rats were examined using commercial ELISA kits purchased from Nanjing Jiancheng Bioengineering Institute. The examinations were performed according to the manufacturer's protocols.

\section{RNA isolation and real-time PCR}

The aortic tissue was homogenized in liquid nitrogen and the total mRNA was isolated using the TaKaRa MiniBEST Universal RNA Extraction Kit (TaKaRa Biotechnology CO., LTD. Dalian, China). Complementary DNA was synthesized from $3 \mu \mathrm{l}$ of total RNA, $0.5 \mu \mathrm{l}$ of oligonucleotide primer $(50 \mu \mathrm{M}), 1 \mu \mathrm{l}$ of super Moloney murine leukemia virus reverse transcriptase (M-MLV) $(200 \mathrm{U} / \mu \mathrm{l})$, $5 \times$ M-MLV buffer, $1.25 \mu \mathrm{l}$ of dNTP mixture, and $0.625 \mu \mathrm{l}$ of RNase inhibitor $(40 \mathrm{U} / \mu \mathrm{l})$ (TaKaRa) in a $25-\mu \mathrm{l}$ reaction system. Quantitative real-time polymerase chain reaction (PCR) was performed using $2 \mu \mathrm{l}$ of cDNA, $1 \mu \mathrm{l}$ of forward and reverse primers (10 $\mu \mathrm{M}$ each), $25 \mu \mathrm{l} 2 \times$ SYBR mix (with $4 \mathrm{mM} \mathrm{Mg}^{2+}$ ) and $0.3 \mu \mathrm{l}$ of Taq DNA polymerase in a $50-\mu \mathrm{l}$ reaction system on a fluorescent quantitative PCR detection system (LineGene 9600, Hangzhou Bioer Technology Co., Ltd., Hangzhou, China). The sequences of primers are presented in Table I.

\section{Western blotting analysis}

The whole protein in aorta was extracted from a total of $20 \mathrm{mg}$ of aortic tissue in each sample using $200 \mu$ l of lysis buffer (Beyotime) on ice. The homogenate was centrifuged at $12,000 \mathrm{~g}$ for $10 \mathrm{~min}$ at $4^{\circ} \mathrm{C}$ and the supernatant was collected. The concentration of protein was determined using the BCA kit (Beyotime). A total of $50 \mu \mathrm{g}$ of proteins in each sample were then separated on $10 \%$ SDS gels and transferred onto polyvinylidene fluoride membranes (Millipore, Billerica, MA, USA). After a blocking stage using 5\% non-fat milk for $1 \mathrm{~h}$ at room temperature, the membranes were incubated with anti-NOX4 ( $1: 500$, Santa Cruz), eNOS (1 : 500, Santa Cruz), LOX-1 (1 : 1000, Abcam, Cambridge, UK), vascular adhesion protein-1 (VAP-1) (1: 500, Santa Cruz), p-65 (1: 1000, Santa Cruz), p-p65 ( $1: 1000$, Santa Cruz) and anti- $\beta$-actin ( $1: 1000$, Santa Cruz) antibodies at $4^{\circ} \mathrm{C}$ overnight. The membranes were then washed in PBS 3 times and incubated with HRP conjugated secondary 
Table I. Primers in real-time PCR

\begin{tabular}{|c|c|c|}
\hline Primer & Forward & Reverse \\
\hline NADPH oxidase type 4 (NOX4) & 5'-TGTTGGGCCTAGGATTGTGT-3' & 5'-CTTCTGTGATCCGCGAAGGT-3' \\
\hline Endothelial nitric oxide synthase (eNOS) & 5'-CTGCCAACGTGGAGATCACT-3' & 5'-GTGAAGAGTTCTGGGGGCTC-3' \\
\hline oxLDL receptor-1 (LOX-1) & 5'-GCCACGGGCTATATCACCTC-3' & 5'-ACATCTGCCCCTCCAGGTAT-3' \\
\hline ICAM-1 & 5'-GCCTGGGGTTGGAGACTAAC-3' & 5'-CTGTCTTCCCCAATGTCGCT-3' \\
\hline$\beta$-actin & 5'-CTCTGTGTGGATTGGTGGCT-3' & 5'-CGCAGCTCAGTAACAGTCCG-3' \\
\hline
\end{tabular}

antibodies (1: 10000, Beyotime) at room temperature for $1 \mathrm{~h}$ and scanned using the Alphalmager HP fluorescent and visible light gel imaging system (ProteinSimple, San Jose, CA, USA). The grey value was analyzed using IPP6 software.

\section{Statistical analysis}

All the data are presented as mean \pm standard deviation (SD). The significance between groups was analyzed using two-way analysis of variance (ANOVA) followed by the least significant difference (LSD) post hoc test using the SAS 9.2 software. $P<$ 0.05 was considered to be statistically significant.

\section{Results}

Effect of repeated positive acceleration exposure on body weights and lipid metabolism

All the rats survived after 8 weeks. The lipid metabolism in the serum was tested following 8 weeks of HFD treatment and $+G$ exposure. As shown in Table II, in the HFD group, serum TC and LDL-C levels were markedly increased compared with the control group, but no change was observed in serum triglyceride (TG) and HDL-C levels. Repeated $+\mathrm{Gz}$ exposure alone did not affect lipid metabolism in the serum, but significantly increased the serum LDL-C level in HFD rats.

\section{Repeated $+\mathrm{Gz}$ exposure and HFD impaired endothelium-dependent relaxation}

Effects of $+\mathrm{Gz}$ and HFD on endothelium-dependent relaxation were evaluated on rats' thoracic aorta. The isolated aorta rings were pre-contracted with $10^{-7} \mathrm{M} \mathrm{NE}$ and then relaxed with cumulative addition of ACh $\left(10^{-9}\right.$ to $\left.10^{-5} \mathrm{M}\right)$, an endothelium-dependent vasodilator agent. $+\mathrm{Gz}$ exposure did not apparently affect the relaxation induced by ACh. However, HFD feeding for 8 weeks significantly attenuated the relaxation response to ACh. The maximal relaxation response decreased from $82.1 \pm 4.0 \%$ to $66.5 \pm 4.3 \%(p<0.01)$. In addition, this impairment induced by HFD was exacerbated by repeated $+\mathrm{Gz}$ exposure. The maximal relaxation of aorta rings from repeated $+\mathrm{Gz}$ exposed HFD rats was $58.2 \pm 4.2 \%$, significantly lower than that in the HFD rats $(p<0.05)$ (Figure 1 A). HFD, $+\mathrm{Gz}$ exposure, or the combination of the two factors did not change the relaxation response of the aorta rings to SNP, an endothelium-independent vasodilator agent (Figure $1 \mathrm{~B}$ ).

\section{Repeated $+\mathrm{Gz}$ exposure and HFD induced ultrastructure changes of endothelial cells}

Under TEM examination, in the control group, the endothelial cells were well organized, the basement membrane was integrated, and the structure of mitochondria was clearly observed (Figure 2 A). However, the endothelial cells in the repeated $+\mathrm{Gz}$ exposed rats were disorganized and endothelial denudation was observed. Some swollen mitochondria were found as well (Figure 2 B). For the HFD group, shed endothelial cells and vacuolar degeneration were found. The mitochondria were swollen and the mitochondria structure was not obvious (Figure $2 \mathrm{C}$ ). In the repeated $+\mathrm{Gz}$ plus HFD group, shed endothelial cells were increased, the basement membranes were damaged, the mi-

Table II. Effects of $+\mathrm{Gz}$ exposure on serum lipid in HFD rats

\begin{tabular}{|lcccc|}
\hline Parameter & Control $(n=8)$ & $\mathbf{+ G z}(n=8)$ & HFD $(n=8)$ & +Gz + HFD $(n=8)$ \\
\hline TC $[\mathrm{mM}]$ & $1.80 \pm 0.15$ & $1.84 \pm 0.41$ & $4.66 \pm 1.54^{* *}$ & $4.79 \pm 0.51$ \\
\hline TG $[\mathrm{mM}]$ & $0.90 \pm 0.11$ & $0.86 \pm 0.18$ & $0.87 \pm 0.17$ & $0.85 \pm 0.11$ \\
\hline HDL-C [mM] & $1.32 \pm 0.13$ & $1.30 \pm 0.35$ & $1.20 \pm 0.38$ & $1.10 \pm 0.25$ \\
\hline LDL-C [mM] & $0.30 \pm 0.02$ & $0.30 \pm 0.06$ & $0.55 \pm 0.16^{\text {** }}$ & $0.75 \pm 0.12^{\# \#}$ \\
\hline
\end{tabular}

Data are expressed as mean $\pm S D$. ${ }^{* *} P<0.01$ compared with control group, ${ }^{\# \#} p<0.01$ compared with HFD group. + Gz - positive acceleration, HFD - high-fat diet, TC - total cholesterol, TG - triglyceride, HDL-C - high-density lipoprotein cholesterol, LDL-C - low-density lipoprotein cholesterol. 
A

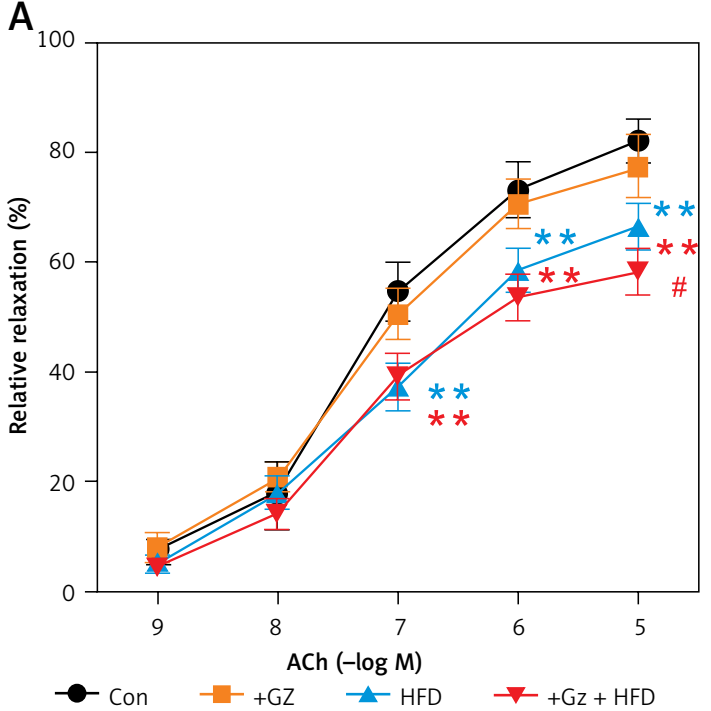

B

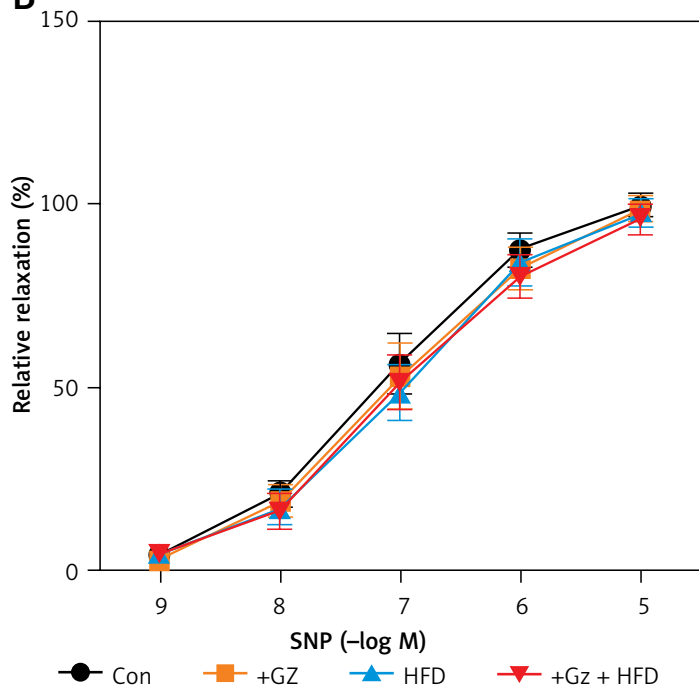

Figure 1. Effects of $+\mathrm{Gz}$ exposure and HFD on the aorta responsiveness to ACh and SNP. A - HFD attenuated ACh-induced vasodilation and $+\mathrm{Gz}$ further decreased the maximal relaxation. $\mathbf{B}-$ Neither $+\mathrm{Gz}$ nor HFD affected SNP-induced vasodilation

Data are expressed as mean $\pm S D . N=8$. ${ }^{* *} P<0.01$ compared with Con group, ${ }^{*} p<0.05$ compared with HFD group.

A

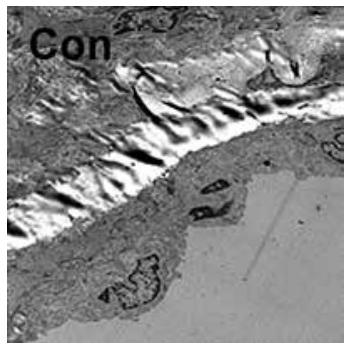

C

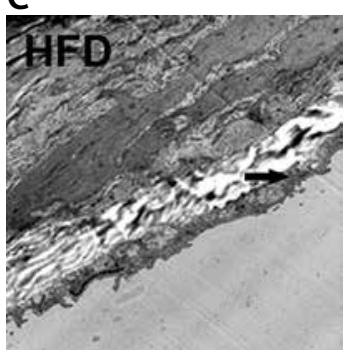

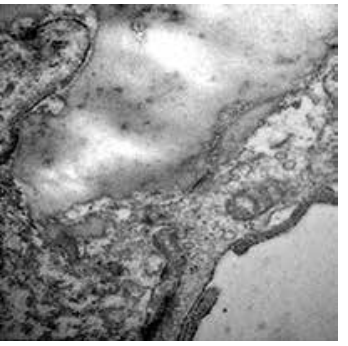

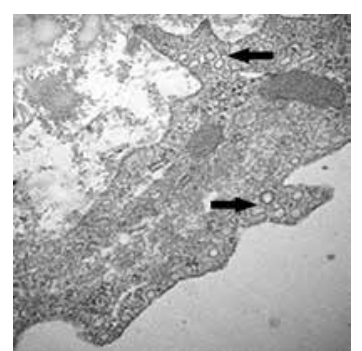

B

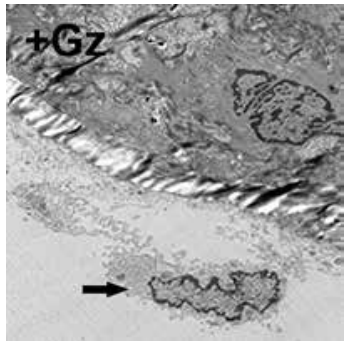

D

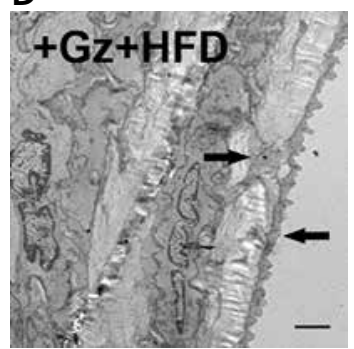

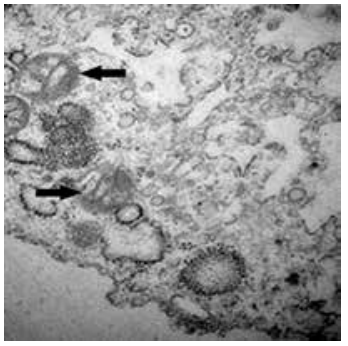

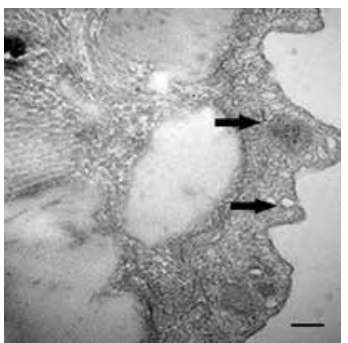

Figure 2. Effects of $+\mathrm{Gz}$ exposure and HFD on ultrastructure of endothelial cells in rat thoracic aorta. A - Control group: normal structure of endothelial cells. B - Repeated $+\mathrm{Gz}$ exposure group: endothelial denudation and swelling of mitochondria were seen. C - HFD group: shedding of endothelial cells and vacuolar degeneration were found. D - Repeated +Gz exposure plus HFD group: increased shedding of endothelial cells, swelling of mitochondria and damaged basement membranes were seen. The scale bar in the left panel in D represents $2 \mu \mathrm{m}$ and the right one represents $100 \mathrm{~nm}$. Ultrastructural changes are indicated by arrows

tochondria was swollen and the crests were dissolved, and cell debris accumulated (Figure 2 D).

\section{Effect of repeated $+\mathrm{Gz}$ exposure and HFD on NO production}

The HFD or $+\mathrm{Gz}$ exposure alone decreased NO level in the thoracic aorta but it was not significant $(p>0.05$, Figure $3 \mathrm{~A})$. However, HFD rats that were repeatedly exposed to $+\mathrm{Gz}$ showed a significantly reduced NO level in the thoracic aorta. In addition, mRNA and protein expression of eNOS were detected as well. The expression of eNOS was dramatically diminished in the $+\mathrm{Gz}+\mathrm{HFD}$ group, which was significantly lower than that in the $+\mathrm{Gz}$ and HFD groups (Figures $3 \mathrm{~B}, \mathrm{C}$ ).

\section{Effect of repeated $+\mathrm{Gz}$ exposure and HFD on oxidative stress}

Markedly reduced T-AOC was observed in $+\mathrm{Gz}$ and HFD groups compared with the control group 

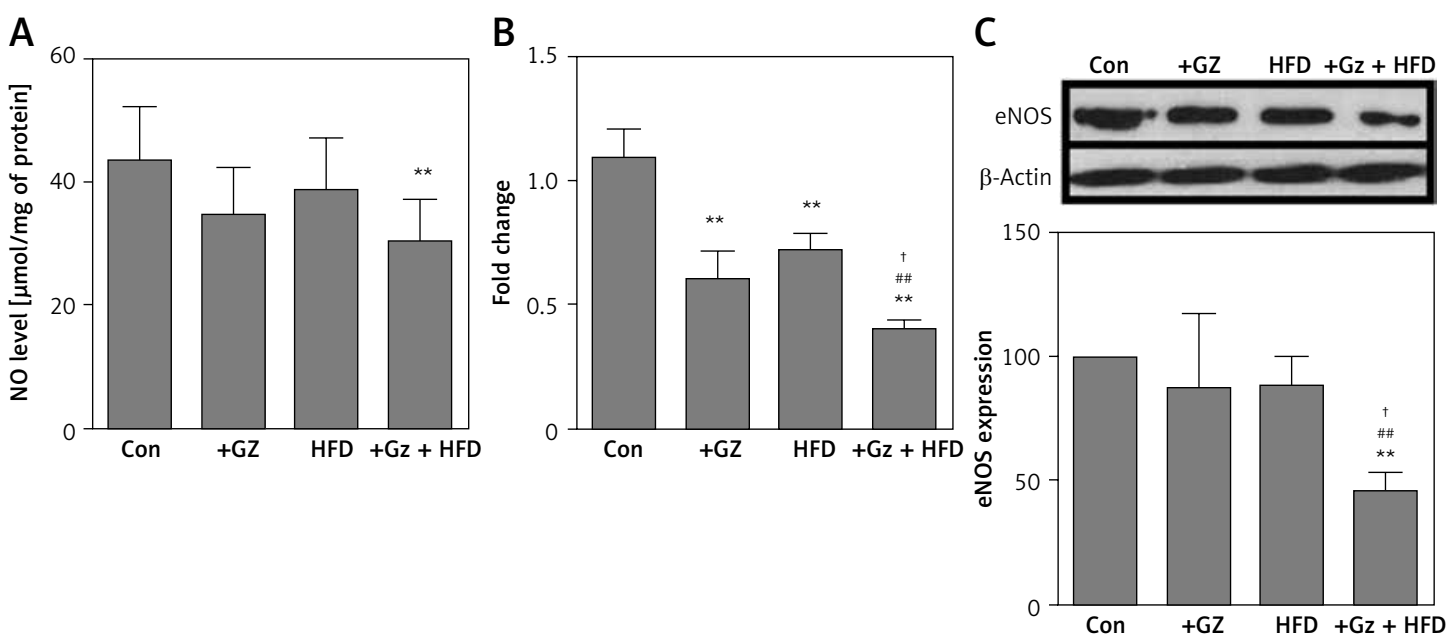

Figure 3. Effects of $+\mathrm{Gz}$ exposure and HFD on NO production in rat thoracic aorta. A - NO content in the aorta. B - mRNA expression of eNOS in the aorta. C - Protein expression of eNOS in the aorta detected using Western blotting

Data are expressed as mean $\pm S D . N=8 .{ }^{* *} P<0.01$ compared with Con group, ${ }^{*} p<0.05$ compared with HFD group, ${ }^{* \# p} p 0.01$ compared with HFD group, ${ }^{\dagger} p<0.05$ compared with $+G z$ group.

A

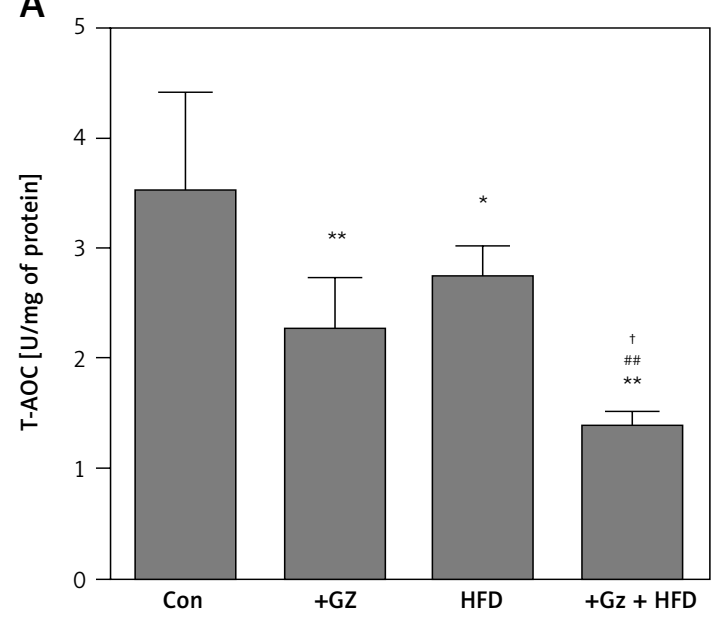

C

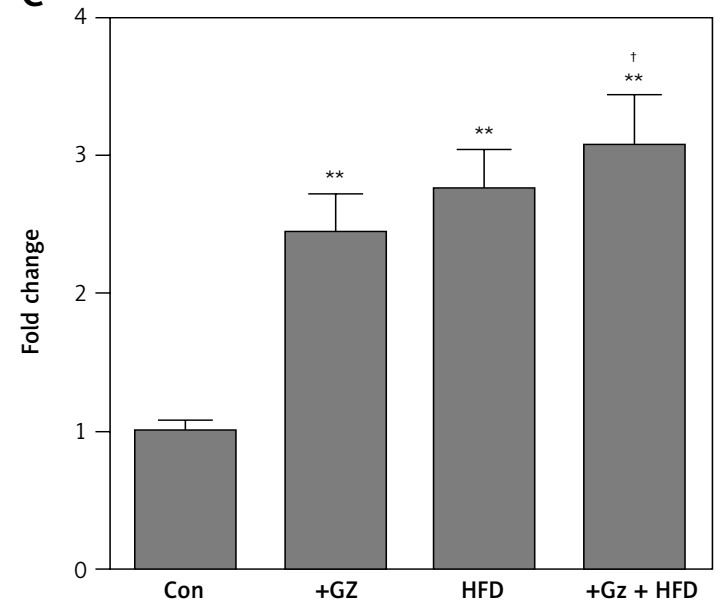

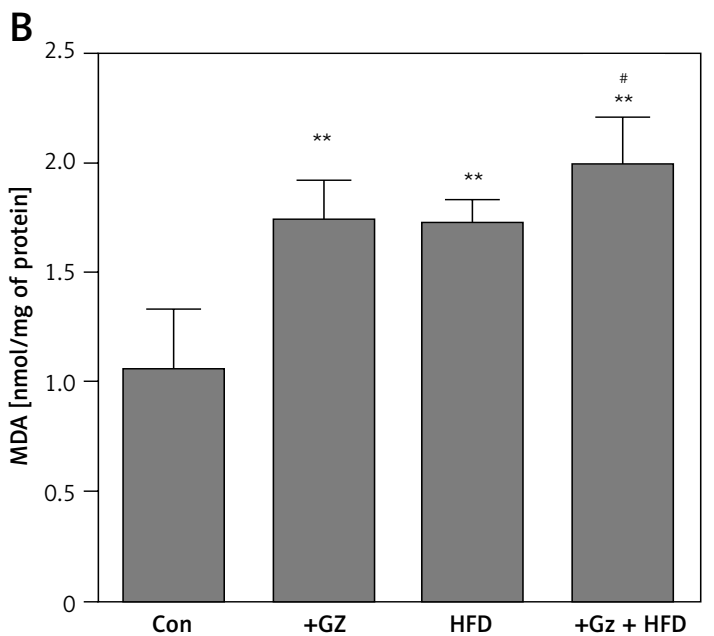

D

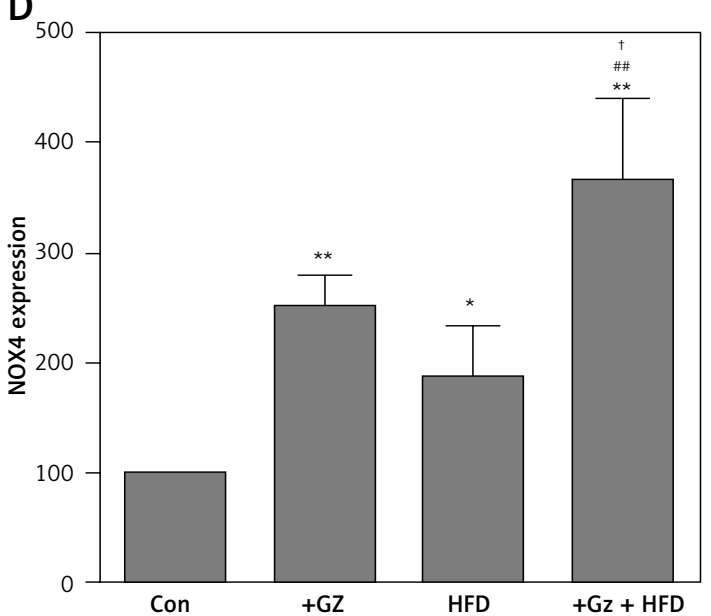

Figure 4. Effects of $+\mathrm{Gz}$ exposure and HFD on T-AOC (A), MDA content (B), and mRNA (C) and protein (D) expression of NOX4 in rat thoracic aorta

Data are expressed as mean $\pm S D . N=8 .{ }^{* *} p<0.01$ compared with Con group, ${ }^{*} p<0.05$ compared with Con group, ${ }^{*} p<0.05$ compared with HFD group, ${ }^{\dagger} p<0.05$ compared with $+G z$ group. 
(Figure $4 \mathrm{~A}$ ). In the $+\mathrm{Gz}+\mathrm{HFD}$ group, the value of T-AOC was much lower than that in $+\mathrm{Gz}$ and HFD groups. Meanwhile, the level of MDA, a marker of oxidative stress, was significantly increased in $+\mathrm{Gz}$ and HFD groups compared with the control group (Figure $4 \mathrm{~B}$ ), and was further increased in the $+\mathrm{Gz}$ plus HFD group. These findings indicate that $+\mathrm{Gz}$ can induce oxidative stress in the aorta and can exacerbate it in HFD rats. NADPH oxidase is the primary source of reactive oxygen species (ROS) in the vasculature [20]. NOX4 is one of the important catalytic subunits of NADPH oxidase. The results of quantitative real-time PCR and Western blotting analysis showed that repeated $+\mathrm{Gz}$ exposure and HFD induced upregulation of NOX4 in the aorta, and the highest levels of NOX4 mRNA and protein expression were seen in the $+\mathrm{Gz}+\mathrm{HFD}$ group (Figures 4 C, D).

\section{Effect of repeated $+\mathrm{Gz}$ exposure and HFD on ox-LDL/LOX-1 expression}

As illustrated in Figures $5 \mathrm{~A}$ and $\mathrm{B}$, repeated $+\mathrm{Gz}$ exposure or HFD caused increases of various magnitude in the serum level of ox-LDL and mRNA and protein expression of LOX-1 in the thoracic aorta, whereas repeated $+\mathrm{Gz}$ exposure plus HFD induced the highest levels of these indexes. oxLDL and LOX-1 lead to endothelial dysfunction through various pathways. In the present study, we also found that repeated $+\mathrm{Gz}$ exposure and HFD induced NF- $\kappa B$ p65 activation (Figure $5 \mathrm{C}$ ). In the $+\mathrm{Gz}+\mathrm{HFD}$ group, the strongest expression of p-p65 was found.

The IL- 6 content in the aorta, mRNA expression of ICAM-1, serum SICAM-1 level and protein expression of VAP-1 were markedly increased in repeated $+\mathrm{Gz}$ exposure and HFD groups, and in line with the results of ox-LDL and LOX-1 expression, repeated $+\mathrm{Gz}$ exposure plus HFD induced the highest levels of these indexes (Figures 5 D-F).

\section{Discussion}

Although the dramatic effects of $+\mathrm{Gz}$ exposure on the cardiovascular system have been described in clinical and experimental studies [17-19], the mechanisms that convert $+\mathrm{Gz}$ stress into physiological dysfunction and changes of molecular expression and functions are much less well known. In the present study, we investigated the effects of $+\mathrm{Gz}$ exposure on endothelial function in normal rats and HFD rats. The results demonstrated that HFD induced endothelial dysfunction, representing by the attenuation of endothelium-dependent relaxation in the rat thoracic aorta, and $+\mathrm{Gz}$ exposure exacerbated the endothelial dysfunction induced by HFD.

Rats, rabbits, and swine have been used in studies of the effects of $+G z$ exposure on the car- diovascular system $[11,19,20]$. Rats are susceptive to HFD and the experiments are easy to perform. Therefore, based on the previous report and the experience in our lab, HFD rats were used to evaluate the effect of $+G z$ exposure on endothelial function in the present study. After 8 weeks HFD feeding and repeated $+\mathrm{Gz}$ exposure, serum TC and LDL-C significantly increased in the HFD group, and the serum LDL-C level was further increased when HFD was combined with $+\mathrm{Gz}$ exposure. The fact that not all the serum lipid indexes were changed in HFD rats may be due to the relatively short HFD feeding period. In order to observe the typical symptoms of AS, most of the studies may extend the HFD feeding period to 16-36 weeks. In our study, we aimed to observe the effect of $+\mathrm{Gz}$ on the development of AS in HFD rats. Therefore, we selected the early stage of AS. At this time point, we found that $+\mathrm{Gz}$ had not affected serum lipid in normal rats, but had increased the LDL-C level in HFD rats. These findings conflict with Luo's study in rabbits [11], in which the effect of $+\mathrm{Gz}$ exposure on lipid was found as early as the $4^{\text {th }}$ week. We considered that this may be due to the differences in the species, experimental methods and the content of cholesterol in the diet. Although we observed only the early stage of AS, the findings indicated that $+\mathrm{G} z$ may be involved in the development of AS in HFD rats.

Nitric oxide is a crucial endothelium-derived molecule that regulates vascular relaxation. The reduction in metabolism and biological activity of NO is a key event of endothelial dysfunction, which contributes to atherosclerosis [21]. In the vascular system, the production of NO is mediated by eNOS. In the present study, we found that the NO level was decreased in the aorta, as well as the mRNA and protein expression of eNOS. In addition, the effects of $+\mathrm{Gz}$ exposure plus HFD were more obvious than those in the treatment of any factor alone. These results suggest that the abnormal NO production contributes to the endothelial dysfunction induced by $+\mathrm{Gz}$ exposure plus HFD, and repeated $+\mathrm{Gz}$ exposure exacerbates the impairment of NO production in HFD rats.

Excessive oxidative stress is an essential pathogenetic feature of atherosclerosis. It is involved in the initiation and progression of the disease and even in the development of complications [22]. Oxidative stress also causes endothelial dysfunction [23]. In vascular tissues, excess superoxide reacts with NO causing reduction of NO bioactivity and reduces eNOS expression and activity in endothelial cells [24]. We found decreased T-AOC and an increased level of MDA, a biomarker of lipid peroxidation, in the aorta of $+\mathrm{Gz}$ and HFD exposed rats, which indicated the imbalance of the antioxidant and oxidant in the vascular tissues. NOX4 

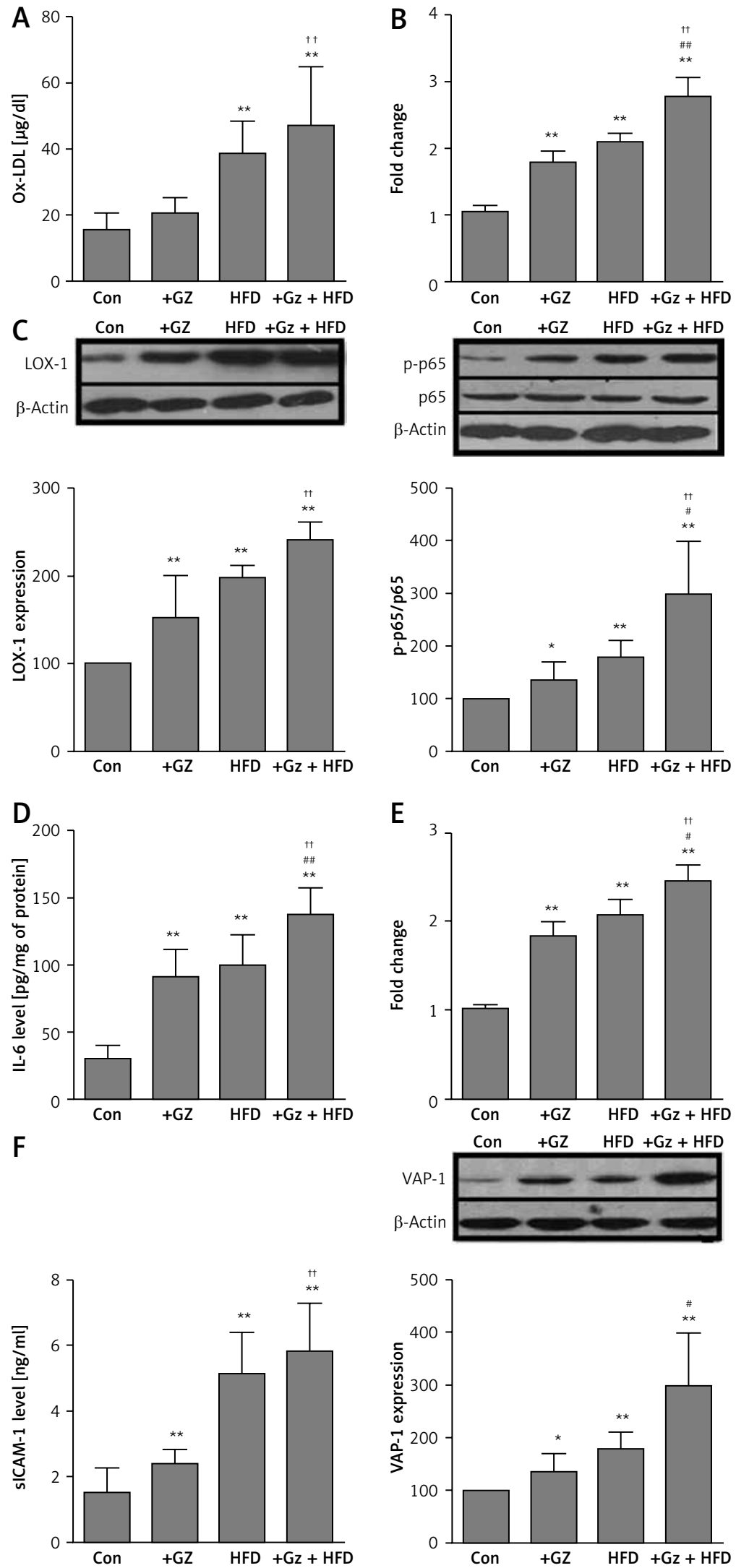

Figure 5. Effects of +Gz exposure and HFD on ox-LDL/LOX-1 expression and inflammatory responses in rat thoracic aorta. A - Serum ox-LDL level. B - mRNA and protein expression of LOX-1 in the aorta. C - Expression levels of NF-KB p-65 and p-p65 in the aorta. D - IL-6 content in the aorta. E - ICAM-1 mRNA expression in the aorta and serum SICAM-1 level. $\mathrm{F}-\mathrm{VAP}$ protein expression in the aorta

Data are expressed as mean $\pm S D . N=8 .{ }^{*} P<0.05$ compared with Con group, ${ }^{* *} p<0.01$ compared with Con group, ${ }^{\#} p<0.05$ compared with HFD group, ${ }^{\# \#} p<0.01$ compared with HFD group, ${ }^{+1} p<0.01$ compared with $+G z$ group. 
is a major isoform of NADPH oxidase expressed in endothelial cells and therefore the main source of ROS in the vasculature [25]. Both the $+\mathrm{Gz}$ exposure and HFD caused increased expression of NOX4 in the aorta, whereas the combination of the two factors induced the highest level of NOX4. This result is consistent with the results of T-AOC and MDA content, and indicates that $+\mathrm{Gz}$ exposure may exacerbate the oxidative stress induced by HFD. The decreased NO level and eNOS expression may, at least partly, result from the oxidative stress in the vasculature.

The oxidative modification of LDL is a crucial factor that promotes the development of atherosclerosis [26]. LOX-1 is the main oxLDL receptor in endothelial cells. LOX-1 is undetectable under normal conditions. It is upregulated when exposed to proatherogenic stimuli such as oxLDL [27]. Ox-LDL/ LOX-1 is associated with various pathological processes in the development of AS including oxidative stress and inflammatory responses. NADPH oxidase has been demonstrated to be involved in oxidation of LDL [28-30], and the expression of LOX-1 can also be induced by proinflammatory cytokines [31]. In the process of AS, ox-LDL/LOX-1 is the primary factor that mediates endothelial dysfunction [32]. ox-LDL/LOX-1 mediates upregulating endothelial adhesion molecules, activating the NF- $\kappa B$ signaling pathway and inducing expression of inflammatory cytokines [33]. In our findings, serum ox-LDL level and the expression of LOX-1 in the aorta were increased in $+G z$ exposed HFD rats compared with those in the HFD rats. The increased ox-LDL may be, at least partly, due to the elevated serum LDL level, because it is derived from circulating LDL. The underlying mechanism for LDL elevation is unclear and is under investigation presently. In addition, the phosphorylated

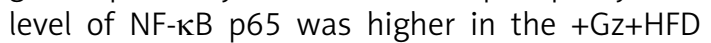
group than in the HFD rats, and the downstream inflammatory cytokines and vasoactive factors were also upregulated. These findings indicate that repeated $+\mathrm{Gz}$ exposure induces AS through enhancing ox-LDL production and LOX-1 expression, and these changes may accelerate the development of HFD-induced endothelial dysfunction, which can help to determine the mechanisms of metabolic changes.

In conclusion, repeated $+G z$ exposure alone can induce endothelial dysfunction in rats, and exposure of the HFD rats to repeated $+\mathrm{Gz}$ exacerbates the dysfunction. The underlying mechanisms include impaired NO production, oxidative stress, abnormal ox-LDL/LOX-1 expression and its downstream molecules. Our observations indicate that repeated $+\mathrm{Gz}$ exposure may increase the risk of AS and also may promote the development of AS.

\section{Conflict of interest}

The authors declare no conflict of interest.

\section{References}

1. Green ND, Ford SA. G-induced loss of consciousness: retrospective survey results from 2259 military air crew. Aviat Space Environ Med 2006; 77: 619-23.

2. Nishida Y, Maruyama S, Shouji I, et al. Effects and biological limitations of $+\mathrm{Gz}$ acceleration on the autonomic functions-related circulation in rats. J Physiol Sci 2016; 66: 447-62.

3. Kim HS, Kim YW. Expression of phosphorylated extracellular signal-regulated kinase in rat kidneys exposed to high +Gz. Bosn J Basic Med Sci 2012; 12: 269-72.

4. Li Y, Zhang T, Deng L, et al. Acquisition technology research of EEG and related physiological signals under +Gz acceleration. Ir J Med Sci 2014; 183: 187-97.

5. Burns JW, Ivan DJ, Stern CH, et al. Protection to $+12 \mathrm{Gz}$. Aviat Space Environ Med 2001; 72: 413-21.

6. Sukhoterin AF, Pashchenko PS. Effects of $+\mathrm{Gz}$ loads on structural organization of central autonomic nuclei. Bull Exp Biol Med 2015; 159: 670-4.

7. Stevenson AT, Scott JP, Chiesa S, et al. Blood pressure, vascular resistance, and $+\mathrm{Gz}$ tolerance during repeated $+\mathrm{Gz}$ exposures. Aviat Space Environ Med 2014; 85: 536-42.

8. Kusiak A, Wiliński J, Wojciechowska W, et al. Echocardiographic assessment of right ventricular function in responders and non-responders tocardiac resynchronization therapy. Arch Med Sci 2015; 11: 736-42.

9. Liao H, Li S, Wei Z, et al. Insights into high-efficiency lignocellulolytic enzyme production by Penicillium oxalicum GZ-2 induced by a complex substrate. Biotechnol Biofuels 2014; 7: 162.

10. Geerling JJ, Boon MR, Kooijman S, et al. Sympathetic nervous system control of triglyceride metabolism: novel concepts derived from recent studies. J Lipid Res 2014; 55: 180-9.

11. Luo H, Chen Y, Wang J. Effects of positive acceleration on the metabolism of endogenous carbon monoxide and serum lipid in atherosclerotic rabbits. J Cardiovasc Dis Res 2010; 1: 75-80.

12. Gimbrone MA Jr, Garcia-Cardena G. Endothelial cell dysfunction and the pathobiology of atherosclerosis. Circ Res 2016; 118: 620-36.

13. Bonetti PO, Lerman LO, Lerman A. Endothelial dysfunction: a marker of atherosclerotic risk. Arterioscler Thromb Vasc Biol 2003; 23: 168-75.

14. Lerman A, Burnett JC Jr. Intact and altered endothelium in regulation of vasomotion. Circulation 1992; 86: III12-9.

15. Yang Z, Zhao A, Li Z, et al. Metabolomics reveals positive acceleration(+Gz)-induced metabolic perturbations and the protective effect of Ginkgo biloba extract in a rat model based on ultra high-performance liquid chromatography coupled with quadrupole time-of-flight mass spectrometry. J Pharm Biomed Anal 2016; 125: 77-84.

16. Chen LE, Wu F, Xin Y, et al. Effect of high sustained $+G z$ stress on myocardial mitochondrial ultrastructure, respiratory function, and antioxidant capacity in rats. J Physiol Sci 2013; 63: 457-64.

17. Fong KL, Fan SW. An overview of the physiological effects of sustained high $+\mathrm{Gz}$ forces on human being. Ann Acad Med Singapore 1997; 26: 94-103.

18. Ozturk C, Ilbasmis MS, Akin A. Cardiac responses to long duration and high magnitude $+\mathrm{Gz}$ exposure in pilots: an 
observational study. Anadolu Kardiyol Derg 2012; 12: 668-74.

19. Xu Y, Li BH, Zhang LH, et al. A centrifuge simulated pushpull manoeuvre with subsequent reduced $+\mathrm{Gz}$ tolerance. Eur J Appl Physiol 2012; 112: 2625-30.

20. Zhang H, Luo H, Sun J, et al. Mild coronary artery stenosis has no impact on cardiac and vascular parameters in miniature swine exposed to positive acceleration stress. J Cardiovasc Med (Hagerstown) 2016; 17: 713-8.

21. Higashi Y, Noma K, Yoshizumi M, et al. Endothelial function and oxidative stress in cardiovascular diseases. Circ J 2009; 73: 411-8.

22. Victor VM, Rocha M, Sola E, et al. Oxidative stress, endothelial dysfunction and atherosclerosis. Curr Pharm Des 2009; 15: 2988-3002.

23. Triggle CR, Howarth A, Cheng ZJ, et al. Twenty-five years since the discovery of endothelium-derived relaxing factor (EDRF): does a dysfunctional endothelium contribute to the development of type 2 diabetes? Can J Physiol Pharmacol 2005; 83: 681-700

24. Harrison D, Griendling KK, Landmesser U, et al. Role of oxidative stress in atherosclerosis. Am J Cardiol 2003; 91: 7A-11A.

25. Ago T, Kitazono T, Ooboshi H, et al. Nox4 as the major catalytic component of an endothelial $\mathrm{NAD}(\mathrm{P}) \mathrm{H}$ oxidase. Circulation 2004; 9: 227-33.

26. Peluso I, Morabito G, Urban L, et al. Oxidative stress in atherosclerosis development: the central role of LDL and oxidative burst. Endocr Metab Immune Disord Drug Targets 2012; 12: 351-60.

27. Gao S, Geng YJ. LOX-1: a male hormone-regulated scavenger receptor for atherosclerosis. Vascul Pharmacol 2013; 59: 138-43.

28. Wang C, Zhao P, Luo P, et al. Prevalence and risk factors of coronary artery disease in patients with chronic viral hepatitis. Postgrad Med 2015; 127: 786-90.

29. Rueckschloss U, Duerrschmidt N, Morawietz H. NADPH oxidase in endothelial cells: impact on atherosclerosis. Antioxid Redox Signal 2003; 5: 171-80.

30. Siqueira ER, Pereira LB, Stefano JT, et al. Association of a variant in the regulatory region of NADPH oxidase 4 gene and metabolic syndrome in patients with chronic hepatitis C. Eur J Med Res 2015; 20: 45.

31. Pirillo A, Reduzzi A, Ferri N, et al. Upregulation of lectin-like oxidized low-density lipoprotein receptor-1 (LOX-1) by 15-lipoxygenase-modified LDL in endothelial cells. Atherosclerosis 2011; 214: 331-7.

32. Pirillo A, Norata GD, Catapano AL. LOX-1, OxLDL, and atherosclerosis. Mediators Inflamm 2013; 2013: 152786.

33. Di Pietro N, Formoso G, Pandolfi A. Physiology and pathophysiology of oxLDL uptake by vascular wall cells in atherosclerosis. Vascul Pharmacol 2016; 84: 1-7. 